\title{
Polymyxin B-immobilized Fiber Column (PMX) Treatment for Idiopathic Pulmonary Fibrosis with Acute Exacerbation: A Multicenter Retrospective Analysis
}

\author{
Shinji Abe ${ }^{1}$, Arata Azuma ${ }^{1}$, Hiroshi Mukae ${ }^{2}$, Takashi Ogura ${ }^{3}$, Hiroyuki Taniguchi ${ }^{4}$, \\ Masashi Bando ${ }^{5}$ and Yukihiko Sugiyama ${ }^{5}$
}

\begin{abstract}
Objective The prognosis of idiopathic pulmonary fibrosis (IPF) patients with acute exacerbation (AE) is reported to be extremely poor. Several clinical studies suggest that direct hemoperfusion with polymyxin Bimmobilized fiber (PMX) may have beneficial effects on AE in patients with interstitial pneumonia (IP). The aim of this multicenter retrospective analysis was to investigate whether PMX treatment could provide improvement of oxygenation and survival benefits in IPF patients with AE.

Methods We conducted a retrospective study of 160 IP patients (including 73 IPF) with AE treated by PMX at 18 institutions in Japan. PMX treatment was carried out twice. The total hemoperfusion time of PMX treatment was, on average, 12 hours. Data concerning oxygenation on PMX treatment and survival after AE were collected and analyzed.

Results In IPF patients with AE, arterial oxygen tension $\left(\mathrm{PaO}_{2}\right) /$ inspiratory oxygen fraction $\left(\mathrm{FiO}_{2}\right),(\mathrm{P} / \mathrm{F}) \mathrm{ra}-$ tio was significantly improved at the end of the 2nd treatment with PMX (173.9 \pm 105.4 to $195.2 \pm 106.8$ Torr, $\mathrm{p}=0.003)$. White blood cell count was significantly reduced at the end of the 2 nd treatment $(13,330 \pm 7,002$ to $\left.9,426 \pm 5,188 / \mathrm{mm}^{3}, \mathrm{p}<0.001\right)$. These clinical changes were also observed on analysis of all 160 IP patients with AE. The one- and three-month survival rates of IPF patients after AE were $70.1 \%$ and $34.4 \%$, respectively.

Conclusion PMX treatment may improve oxygenation and survival in IPF patients with AE. Prospective, controlled trials of PMX treatment for IPF with AE are warranted to verify this potential benefit.
\end{abstract}

Key words: interstitial pneumonia (IP), idiopathic pulmonary fibrosis (IPF), acute exacerbation, polymyxin B-immobilized fiber column (PMX)

(Intern Med 51: 1487-1491, 2012)

(DOI: 10.2169/internalmedicine.51.6965)

\section{Introduction}

Idiopathic pulmonary fibrosis (IPF), the most common form of idiopathic interstitial pneumonia (IIP), is characterized by clinically progressive dyspnea with worsening respiratory function $(1,2)$. The prognosis of IPF has been reported to be poor with a mean survival of approximately 3 years after diagnosis $(1,3)$. Acute exacerbation (AE) of IPF (AE-IPF) has been increasingly recognized as a common and critical event in the clinical course of IPF (4-9). AE-IPF is generally accompanied by progressive respiratory failure and pathologically diffuse alveolar damage (DAD) superimposed on usual interstitial pneumonia (UIP) (4-8). Current therapies including high-dose corticosteroid, antiinflammatory and immunosuppressive agents provide little

\footnotetext{
${ }^{1}$ Internal Medicine, Department of Pulmonary Medicine/Infection and Oncology, Nippon Medical School, Japan, ${ }^{2}$ Department of Respiratory Medicine, University of Occupational and Environmental Health, Japan, ${ }^{3}$ Department of Respiratory Medicine, Kanagawa Cardiovascular and Respiratory Center, Japan, ${ }^{4}$ Department of Respiratory Medicine, Tosei General Hospital, Japan and ${ }^{5}$ Division of Pulmonary Medicine, Department of Medicine, Jichi Medical University, Japan

Received for publication November 18, 2011; Accepted for publication March 6, 2012
}

Correspondence to Dr. Shinji Abe, sabe@nms.ac.jp 
Table 1. Patient Characteristics at Pretreatment Baseline

\begin{tabular}{|c|c|c|}
\hline Characteristics & all patients $(n=160)$ & $\operatorname{IPF}(n=73)$ \\
\hline Age (years) & $67.1 \pm 8.5(40-85)$ & $67.5 \pm 8.2(47-84)$ \\
\hline Male/Female (n) & $111 / 49$ & $58 / 15$ \\
\hline Smoking index & $880 \pm 633$ & $937 \pm 658$ \\
\hline$\% \mathrm{VC}(\%)$ & $73.7 \pm 19.5(\mathrm{n}=78)$ & $70.7 \pm 18.1(\mathrm{n}=48)$ \\
\hline \multicolumn{3}{|l|}{ Underlying disease (n) } \\
\hline IIPs & 108 & \\
\hline CVD-IP & 30 & \\
\hline Drug-induced IP & 7 & \\
\hline RPIP of unknown etiology & 5 & \\
\hline CHP & 5 & \\
\hline Others & 5 & \\
\hline \multicolumn{3}{|c|}{$\begin{array}{l}\text { Data are given as mean } \pm \text { SD, IPF: idiopathic pulmonary fibrosis, IIPs: idiopathic interstitial } \\
\text { pneumonias, CVD-IP: collagen vascular disease related interstitial pneumonia, IP: interstitial } \\
\text { pneumonia, RPIP: rapidly progressive interstitial pneumonia, CHP: chronic hypersensitivity } \\
\text { pneumonia }\end{array}$} \\
\hline
\end{tabular}

Table 2. Clinical Status of Patients at PMX Treatment

\begin{tabular}{lcc}
\hline & all patients $(\mathrm{n}=160)$ & IPF $(\mathrm{n}=73)$ \\
\hline $\begin{array}{l}\text { Number of treatments }(\mathrm{n}) \\
\text { Total perfusion time }(\mathrm{h})\end{array}$ & 2.0 & 2.0 \\
$\begin{array}{l}\text { Interval between } \\
1^{\text {st }} \text { and 2nd } \text { PMX (h) }\end{array}$ & $12.5 \pm 10.0$ & $12.4 \pm 9.3$ \\
& & \\
Anticoagulant & & $13.9 \pm 9.0$ \\
$\quad$ Nafamostat mesilate & $113(77.4 \%)$ & $51(73.9 \%)$ \\
$\quad$ Heparin sodium & $32(21.9 \%)$ & $15(21.7 \%)$ \\
$\quad$ dalteparin sodium & $6(4.1 \%)$ & $5(7.2 \%)$ \\
Steroid-pulse therapy & $160(100 \%)$ & $73(100 \%)$ \\
$\quad$ before PMX & $157(98.1 \%)$ & $72(98.6 \%)$ \\
$\quad$ after PMX & $3(1.9 \%)$ & $1(1.4 \%)$ \\
\hline Data are given as mean \pm SD & &
\end{tabular}

benefit for IPF patients with AE (5-9). It has recently been reported that acute exacerbations are not unique to IPF patients, but also occur in other fibrotic lung diseases such as non-specific interstitial pneumonia (NSIP) or collagen vascular disease-related interstitial pneumonia (CVD-IP) (10).

The polymyxin B-immobilized fiber column (PMX) was originally developed for the removal of endotoxin and is used for the treatment of endotoxemia. Several studies have reported an improvement of oxygenation with PMX treatment in acute lung injury/acute respiratory distress syndrome (ALI/ARDS), which is characterized pathologically as DAD (11-13). Recent clinical reports have suggested beneficial effects of PMX treatment on oxygenation in IPF patients with AE (14-16). PMX treatment has been considered to be one of the therapeutic options for acute exacerbation of IP. However, there has been no large-scale evaluation of the effect of PMX treatment in acute exacerbation of interstitial pneumonia (IP) in Japan. This retrospective study was aimed to clarify the effect of PMX treatment on oxygenation and the survival of IP, especially IPF patients with $\mathrm{AE}$.

\section{Materials and Methods}

\section{Patient background}

One hundred and sixty patients from 18 institutions in Japan were studied. The study was approved by each institutional review board. Medical records were reviewed to identify patients with IP, including IPF, who experienced AE and were treated with PMX. IPF was defined according to American Thoracic Society (ATS)/European Respiratory Society (ERS) criteria $(1,2)$. AE-IPF was defined using the revised Japanese criteria for $\operatorname{AE}-\operatorname{IPF}(17,18)$, which state that all of the following three conditions must be satisfied during the course of IPF within a single month: (1) dyspnea increases, (2) new ground-glass opacities appear on HRCT in addition to previous honeycomb lesions, (3) partial pressure of oxygen in resting arterial blood $\left(\mathrm{PaO}_{2}\right)$ is lower by more than 10 Torr than in previous measurements. It is important to exclude obvious causes of these changes, such as infection, pneumothorax, cancer, pulmonary embolism or congestive heart failure. All patients fulfilled the above criteria. All decisions regarding diagnostic evaluation and choice of treatment were independently made by expert clinicians at each institution. Blood endotoxin levels before PMX treatment were below detection levels in all cases.

\section{PMX treatment}

Direct hemoperfusion with PMX (Toraymyxin 20R, Toray Medical Co., Tokyo, Japan) was performed once daily for 2 successive days at a flow rate of $80-100 \mathrm{~mL} / \mathrm{min}$. Blood access for direct hemoperfusion with PMX was obtained by means of a double lumen catheter inserted into the femoral vein according to the Seldinger method.

\section{Statistical analysis}

Statistical analysis was performed using software as follows: paired t-test by Excel 2003, non-paired t-test and other analysis by Statcel 2 (The Publisher OMS Ltd., Tokorozawa, Japan). Data are expressed as the mean \pm standard deviation (SD). Cumulative survival is described using the KaplanMeier method. Differences were considered significant at $\mathrm{p}<$ 0.05 .

\section{Results}

\section{Patient characteristics}

From 2004 to 2008, 160 IP patients (111 males), including 73 IPF (58 males), with AE were enrolled in the multicenter study. The demographic and clinical features are shown in Table 1 and 2. Etiologies other than IPF included CVD-IP ( $n=30)$, drug-induced IP $(n=7)$, rapidly progressive interstitial pneumonia (RPIP) of unknown etiology (19) (n= 5 ) and chronic hypersensitivity pneumonia $(n=5)$. Pulmonary function tests of IPF patients $(\mathrm{n}=48)$ within 3 months before 
Table 3. Effect of PMX on P/F in Interstitial Pneumonia (all and IPF) Patients with Acute Exacerbation

\begin{tabular}{cccc}
\hline & pre-PMX & end of 1st PMX & end of 2nd PMX \\
\hline all patients & $148.9 \pm 87.2$ & $177.7 \pm 108.7^{*}$ & $175.1 \pm 92.5^{*}$ \\
IPF & $173.9 \pm 105.4$ & $205.4 \pm 122.1^{\#}$ & $195.2 \pm 106.8^{\# \#}$ \\
\hline Data are given as mean \pm SD (Torr) \\
${ }^{*}$ p $<0.0001$ compared to pre-PMX \\
\#p=0.001 compared to pre-PMX, \#\#p=0.003 compared to pre-PMX
\end{tabular}

Table 4. Effect of PMX on $\mathrm{PaCO}_{2}$ in Interstitial Pneumonia (all and IPF) Patients with Acute Exacerbation

\begin{tabular}{cccc}
\hline & pre-PMX & end of 1st PMX & end of 2nd PMX \\
\hline all patients & $45.5 \pm 11.7$ & $45.9 \pm 15.8$ & $45.2 \pm 15.8$ \\
IPF & $45.3 \pm 12.3$ & $47.5 \pm 15.9$ & $47.2 \pm 13.9$ \\
\hline
\end{tabular}

Data are given as mean \pm SD $($ Torr $)$

PMX treatment revealed moderate restrictive impairment (\% VC $70.7 \pm 18.1 \%$ of predicted). All 160 patients received high-dose corticosteroid therapy. The majority of patients (98\%) were treated with corticosteroids before PMX. The average number of PMX treatments was two in all IP and in IPF patients. The total hemoperfusion time of PMX treatment was, on average, 12 hours, indicating that each hemoperfusion was performed for approximately 6 hours.

\section{Response to PMX treatment}

As shown in Table 3, the P/F ratio before PMX treatment was low, with averages of 148.9 in all IP patients and 173.9 in IPF. PMX treatment significantly improved P/F ratio at the end of the 2nd hemoperfusion in all IP and IPF cases: $148.9 \pm 87.2$ to $175.1 \pm 92.5$ Torr in all IP patients $(\mathrm{p}<0.0001)$, $173.9 \pm 105.4$ to $195.2 \pm 106.8$ Torr in IPF $(p=0.003)$. There was no significant change of arterial $\mathrm{PaCO}_{2}$ after the two PMX treatments (Table 4). The peripheral white blood cell count decreased significantly in all IP and IPF patients: $13,330 \pm 7,002$ to $9,426 \pm 5,188 / \mathrm{mm}^{3}$ in all IP patients $(\mathrm{p}<$ $0.0001), 13,516 \pm 8,422$ to $9,873 \pm 6,041 / \mathrm{mm}^{3}$ in IPF (Table 5).

\section{Safety of PMX treatment}

During the treatment course, no patient discontinued therapy because of any side effects with PMX use. Twelve of $160(8.0 \%)$ patients had mild thrombocytopenia after PMX treatment. All twelve patients promptly recovered without blood transfusion.

\section{Survival analysis}

Survival rates after exacerbation in IPF patients by Kaplan-Meier analysis reached $70.1 \%$ and $34.5 \%$ at one and three months, respectively. Survival rates were comparable in all 160 IP patients with AE (Fig. 1, 2). To evaluate the
Table 5. Effect of PMX on Peripheral White Blood Cell Count in Interstitial Pneumonia (all and IPF) Patients with Acute Exacerbation

\begin{tabular}{lccc}
\hline & pre-PMX & end of 1st PMX & end of $2^{\text {nd }}$ PMX \\
\hline all patients & $13,330 \pm 7,002$ & $10,466 \pm 7,417^{*}$ & $9,426 \pm 5,188^{*}$ \\
IPF & $13,516 \pm 8,422$ & $10,885 \pm 8,267^{\#}$ & $9,873 \pm 6,041^{\#}$ \\
\hline \multicolumn{2}{c}{ Data are given as mean \pm SD $\left(/ \mathrm{mm}^{3}\right)$} \\
${ }^{*} \mathrm{p}<0.0001$ compared to pre-PMX \\
$\# \mathrm{p}<0.0001$ compared to pre-PMX
\end{tabular}

improvement of oxygenation by PMX treatment contributing to better survival in IP or IPF patients with $\mathrm{AE}$, the patients were divided into 3-month survivors $(n=48$ in all and $n=25$ in IPF patients) and non-survivors. P/F ratios were significantly improved in both 3-month survivors of all IP patients and IPF patients (Table 6). These results indicated that PMX treatment could bring about a better prognosis in IP, including IPF, patients with AE.

\section{Discussion}

This is the first study reporting the effect of PMX treatment on pulmonary oxygenation and survival in a large population of IP patients with AE, including IPF. The results show that PMX treatment significantly improved $\mathrm{P} / \mathrm{F}$ ratio and may provide survival benefit across the whole spectrum of IP with AE, including IPF.

There is no established treatment for AE in IP patients. Empirically, high-dose corticosteroid (500 to $1,000 \mathrm{mg}$ methylprednisolone per day for 3 days), broad-spectrum antibiotics and immunosuppressive agents have often been administered. However, these agents provide little benefit (5-9). Although the majority of patients (98\%) in the present study received high-dose corticosteroids, they were selected for PMX treatment, suggesting that high-dose steroid therapy had little benefit for AE in IP patients.

The prognosis of IPF patients is reportedly poor with high mortality rates of approximately $80 \%$ at one month and $96 \%$ at three months after the diagnosis of acute exacerbation $(5,8,9)$. Huie et al. have recently reported high mortality in patients with not only IPF, but also other fibrotic lung disease, who were admitted with acute respiratory decline (20). In the present study, the survival rates at three months after AE were $30.1 \%$ and $34.5 \%$ in all IP patients and in IPF, respectively. These results suggest that PMX treatment may have therapeutic benefits not only in IPF-AE, but also in other interstitial pneumonias exhibiting acute decline.

Studies of PMX treatment in IP patients with AE have been limited to several retrospective case reports and small case series. Previous reports have suggested the beneficial effect of PMX treatment on pulmonary oxygenation in IP patients with AE. Seo et al. have reported that alveolararterial oxygen difference $\left(\mathrm{AaDO}_{2}\right)$, serum KL-6 and lactate dehydrogenase (LDH) were improved after PMX treatment 


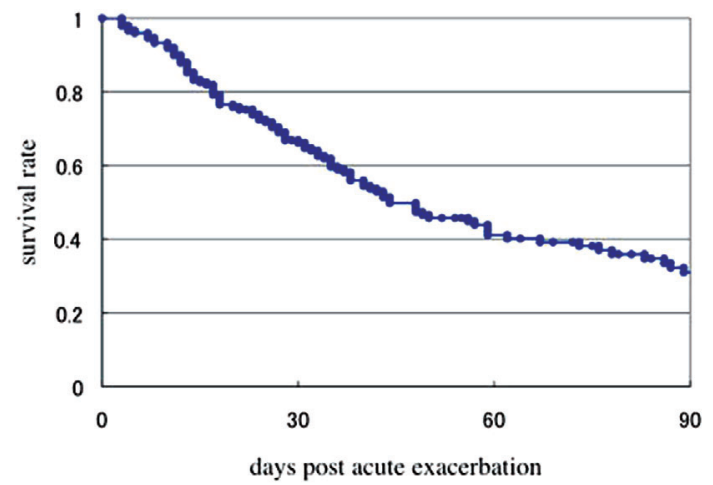

Figure 1. Kaplan-Meier plot of the survival rate from the diagnosis of acute exacerbation for interstitial pneumonia (IP) patients treated with polymyxin B-immobilized fiber column (PMX) (all patients; $\mathrm{n}=160$, censored at 90 days).

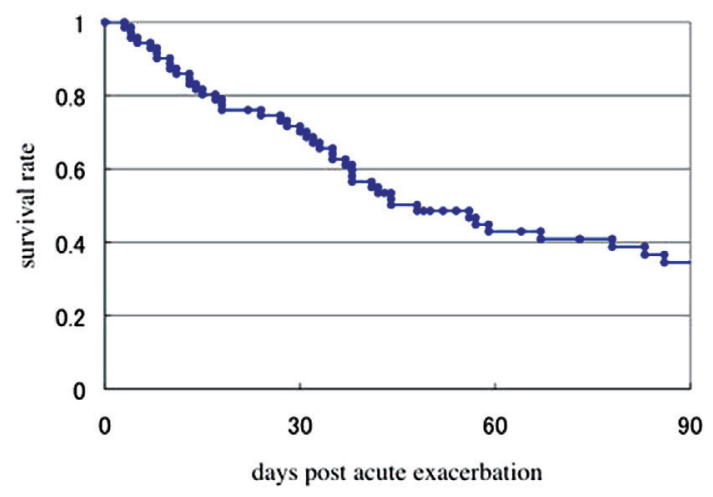

Figure 2. Kaplan-Meier plot of the survival rate from the diagnosis of acute exacerbation for idiopathic pulmonary fibrosis (IPF) patients treated with polymyxin B-immobilized fiber column (PMX) $(n=73$, censored at 90 days).

in four of six IPF patients with AE. These four patients were successfully weaned from mechanical ventilation and survived more than 30 days after the initial PMX treatment (14). Enomoto et al. have reported that PMX treatment improved oxygenation and HRCT findings in five patients with IP, including IPF. They showed that two patients treated with PMX for 12 hours had a better prognosis, suggesting the beneficial effects of longer hemoperfusion with PMX (15). Recently, Hara et al. have reported the effects of PMX treatment in 33 consecutive IP patients with AE who were resistant to steroid pulse therapy. They demonstrated that PMX treatment improved oxygenation at 72 hours and at 1 week after the first PMX treatment. The improvement in oxygenation was observed in both IIP and non-IIP patients (16).

The mechanism whereby PMX treatment exerts beneficial effects in AE-IPF is still uncertain. Beneficial effects of PMX treatment on inflammatory cells or mediators have been recently reported. We have reported that neutrophils absorbed by PMX were highly activated and serum matrix metalloproteinase (MMP)-9 levels were significantly decreased after PMX treatment in IP patients with AE (21).
Table 6. Effect of PMX on $\mathrm{P} / \mathrm{F}$ in Interstitial Pneumonia (all and IPF) Patients with Acute Exacerbation: Comparison of 3-Month-survivors and Non-survivors

\begin{tabular}{clcc}
\hline & & pre-PMX & end of 2nd PMX \\
\hline \multirow{2}{*}{ all patients } & survivors & $167.1 \pm 95.3$ & $208.1 \pm 120.8^{*}$ \\
& non-survivors & $135.0 \pm 83.1$ & $149.5 \pm 71.0$ \\
\multirow{2}{*}{ IPF } & survivors & $166.0 \pm 97.1$ & $216.2 \pm 108.9^{\#}$ \\
& non-survivors & $133.9 \pm 70.9$ & $152.4 \pm 91.5$ \\
\hline
\end{tabular}

Data are given as mean $\pm \mathrm{SD}$

${ }^{*} \mathrm{p}=0.04$ compared to pre-PMX, ${ }^{*} \mathrm{p}=0.03$ compared to pre-PMX

Hara et al. reported that serum levels of monocyte chemotactic protein (MCP)-1 were significantly decreased after PMX treatment, suggesting an inhibitory effect of PMX on monocyte activation (16). In the present study, the number of peripheral white blood cells was significantly reduced after PMX treatment. However, all patients were treated with high-dose corticosteroid and the number of white blood cells might be affected by the concurrent therapies. Further studies are needed to establish to the precise mechanism of PMX treatment in IP or IPF patients with AE.

Our study had several important limitations. The study was retrospective, and the timing and duration of PMX treatment differed in each institution, which may have rendered bias in the results. In addition, data from concurrent therapies including mechanical ventilation and immunosuppressive agents were not evaluated. Therapies or management strategies concurrent with PMX treatment may have influenced the clinical courses. Furthermore, we only analyzed the outcome up to 3 months after $\mathrm{AE}$, and long-term follow-up would be required.

In summary, we found a favorable clinical response to PMX treatment among IP patients, including IPF patients, with AE. Although our sample size was limited, nonetheless it is the largest therapeutic and prognostic study to date in IPF patients with AE. There was a demonstrable benefit in oxygenation of IP or IPF patients with AE. We believe that the results of our study, along with those of previous reports, support the potential utility of PMX treatment in IP or IPF patients with AE. Prospective controlled studies of PMX treatment are needed to confirm this apparent effect.

\section{The authors state that they have no Conflict of Interest (COI).}

\section{Acknowledgement}

This study was supported by the Health and Labor Sciences Research Grants on Diffuse Lung Diseases from the Japanese Ministry of Health, Labor and Welfare. We thank the following institutions for having included patients in the study: Tohoku University Graduate School of Medicine (Miyagi, Japan), National Hospital Organization Ibaraki Higashi Hospital (Ibaraki, Japan), Kyoto-Katsura Hospital (Kyoto, Japan), National Center for Global Health and Medicine (Tokyo, Japan), Tenri Hospital (Nara, Japan), Tokyo Medical and Dental University (Tokyo, Ja- 
pan), Sakai Hospital Kinki University Faculty of Medicine (Osaka, Japan), Tokyo Medical University (Tokyo, Japan), National Hospital Kinki-Chuo Chest Medical Center (Osaka, Japan), Kobe City Medical Center General Hospital (Hyogo, Japan), Nagasaki University School of Medicine (Nagasaki, Japan), Hamamatsu University School of Medicine (Shizuoka, Japan), Oita University Faculty of Medicine (Oita, Japan), Shinshu University School of Medicine (Nagano, Japan), Kagoshima University Graduate School of Medical and Dental Sciences (Kagoshima, Japan).

\section{References}

1. American Thoracic Society/European Respiratory Society International Multidisciplinary Consensus Classification of the Idiopathic Interstitial Pneumonias. This joint statement of the American Thoracic Society (ATS), and the European Respiratory Society (ERS) was adopted by the ATS board of directors, June 2001 and by the ERS Executive Committee, June 2001. Am J Respir Crit Care Med 165: 277-304, 2002 (Erratum in: Am J Respir Crit Care Med 166: 426, 2002).

2. American Thoracic Society. Idiopathic pulmonary fibrosis: diagnosis and treatment. International consensus statement. American Thoracic Society (ATS), and the European Respiratory Society (ERS). Am J Respir Crit Care Med 161: 646-664, 2000.

3. Kim DS, Collard HR, King TE Jr. Classification and natural history of the idiopathic interstitial pneumonias. Proc Am Thorac Soc 3: 285-292, 2006.

4. Kondoh Y, Taniguchi H, Kawabata Y, Yokoi T, Suzuki K, Takagi $\mathrm{K}$. Acute exacerbation in idiopathic pulmonary fibrosis: analysis of clinical and pathological finding in three cases. Chest 103: 1808-1812, 1993

5. Ambrosini V, Cancellieri A, Chilosi M, et al. Acute exacerbation of idiopathic pulmonary fibrosis: report of a series. Eur Respir J 22: 821-826, 2003.

6. Churg AC, Muller NL, Silva CIS, Wright JL. Acute exacerbation (acute lung injury of unknown cause) in UIP and other forms of fibrotic interstitial pneumonias. Am J Surg Pathol 31: 277-284, 2007.

7. Tiitto L, Bloigu R, Heiskanen U, Paakko P, Kinnula VL, Wiik RK. Relationship between histopathological features and the course of idiopathic pulmonary fibrosis/usual interstitial pneumonia. Thorax 61: 1091-1095, 2006.

8. Kim DS, Park JH, Park BK, Lee JS, Nicholson AG, Colby T. Acute exacerbation of idiopathic pulmonary fibrosis: frequency and clinical features. Eur Respir J 27: 143-150, 2006.
9. Al-Hameed FM, Sharma S. Outcome of patients admitted to the intensive care unit for acute exacerbation of idiopathic pulmonary fibrosis. Can Respir J 11: 117-122, 2004.

10. Park IN, Kim DS, Shim TS, et al. Acute exacerbation of interstitial pneumonia other than idiopathic pulmonary fibrosis. Chest 132: 214-220, 2007.

11. Tsushima K, Kubo K, Koizumi T, et al. Direct hemoperfusion using a polymyxin $\mathrm{B}$ immobilized column improves acute respiratory distress syndrome. J Clin Apher 17: 97-102, 2002.

12. Nakamura T, Kawagoe $Y$, Matsuda $T$, et al. Effect of polymyxin B-immoblized fiber on blood metalloproteinase- 9 and tissue inhibitor of metalloproteinase-1 levels in acute respiratory distress syndrome patients. Blood Purif 22: 256-260, 2004.

13. Kushi H, Miki T, Okamoto K, Nakahara J, Saito T, Tanjoh K. Early hemoperfusion with an immobilized polymyxin B fiber column eliminates humoral mediators and improves pulmonary oxygenation. Crit Care 9: R653-R661, 2005.

14. Seo Y, Abe S, Kurahara M, et al. Beneficial effect of polymyxin B-immobilized fiber column (PMX-DHP) hemoperfusion treatment on acute exacerbation of idiopathic pulmonary fibrosis. Results of a pilot study. Intern Med 45: 1033-1038, 2006.

15. Enomoto N, Suda T, Uto T, et al. Possible therapeutic effect of direct hemoperfusion with a polymyxin B immobilized fibre column (PMX-DHP) on pulmonary oxygenation in acute exacerbation of interstitial pneumonia. Respirology 13: 452-460, 2008.

16. Hara S, Ishimoto $\mathrm{H}$, Sakamoto $\mathrm{N}$, et al. Direct hemoperfusion using immobilized polymyxin B in patients with rapidly progressive interstitial pneumonias: a retrospective study. Respiration 81: 107117, 2011.

17. Taniguchi H, Ebina M, Kondoh Y, et al. Pirfenidone Clinical Study Group in Japan. Eur Respir J 35: 821-829, 2010.

18. Taniguchi $\mathrm{H}$, Kondoh $\mathrm{Y}$. Revised criteria for acute exacerbation of idiopathic pulmonary fibrosis. The Annual Report by Study Group of Ministry of Health and Welfare for Diffuse Lung Disease. Diffiuse Lung Diseases Research Group from the Ministry of Health, Labor and Welfare of Japanese Government, 2004: 114-119.

19. Kondoh Y, Taniguchi H, Kataoka K, et al; Tokai Diffuse Lung Disease Study Group. Prognostic factors in rapidly progressive interstitial pneumonia. Respirology 15: 257-264, 2010.

20. Huie TJ, Olson AL, Cosgrove GP, et al. A detailed evaluation of acute respiratory decline in patients with fibrotic lung disease: aetiology and outcomes. Respirology 15: 909-917, 2010.

21. Abe S, Seo Y, Hayashi H, et al. Neutrophil adsorption by polymyxin B-immobilized fiber column for acute exacerbation in patients with interstitial pneumonia: a pilot study. Blood Purif 29: 321-326, 2010.

(C) 2012 The Japanese Society of Internal Medicine http://www.naika.or.jp/imindex.html 